\title{
1 Photorealistic reconstruction of visual texture from EEG signals
}

3 Suguru Wakita ${ }^{1 *}$, Taiki Orima ${ }^{1,2}$, Isamu Motoyoshi $^{1}$

41 Department of Life Sciences, The University of Tokyo, Tokyo, Japan

52 Japan Society for the Promotion of Science, Tokyo, Japan

6 *Corresponding Author:

7 suguruwakita.ac@gmail.com

\section{Abstract}

9 Recent advances in brain decoding have made it possible to classify image categories

10 based on neural activity. Increasing numbers of studies have further attempted to

11 reconstruct the image itself. However, because images of objects and scenes inherently

12 involve spatial layout information, the reconstruction usually requires retinotopically

13 organized neural data with high spatial resolution, such as fMRI signals. In contrast,

14 spatial layout does not matter in the perception of 'texture', which is known to be

15 represented as spatially global image statistics in the visual cortex. This property of

16 'texture' enables us to reconstruct the perceived image from EEG signals, which have a

17 low spatial resolution. Here, we propose an MVAE-based approach for reconstructing

18 texture images from visual evoked potentials measured from observers viewing natural

19 textures such as the textures of various surfaces and object ensembles. This approach

20 allowed us to reconstruct images that perceptually resemble the original textures with a

21 photographic appearance. A subsequent analysis of the dynamic development of the

22 internal texture representation in the VGG network showed that the reproductivity of

23 texture rapidly improves at 200 ms latency in the lower layers but improves more

24 gradually in the higher layers. The present approach can be used as a method for

25 decoding the highly detailed 'impression' of sensory stimuli from brain activity. 


\section{Introduction}

28 In the field of neuroscience, an increasing number of studies have been conducted to

29 estimate perceptual content and psychological states by extracting certain statistical

30 patterns from brain activity data (Kamitani \& Tong, 2005; Schwartz et al., 2006;

31 Miyawaki et al., 2008; Carlson et al., 2011; Green \& Kalaska, 2011; Nishimoto et al.,

32 2011). A number of 'brain decoding' techniques that identify the object category of an

33 image from the fMRI-BOLD signal have been reported (Shenoy \& Tan, 2008; Das et al.,

34 2010; Wang et al., 2012; Carlson et al., 2013; Stewart et al., 2014; Kaneshiro et al.,

35 2015). In recent years, ambitious attempts have been made to reconstruct the image

36 itself from brain activity (Palazzo et al., 2017; Shen et al., 2019a; Shen et al., 2019b).

37 For instance, Shen et al. (Shen et al., 2019a) proposed a method of decoding visual

38 features for each hierarchical stage of visual information processing from an fMRI signal

39 using a deep neural network (DNN) (Krizhevsky et al., 2012; Simonyan \& Zisserman

40 2015, He et al., 2016) and successfully reconstructed not only the presented image but

41 also the image that an observer imagined in her/his mind.

42 While excellent decoding is supported by the big data of fMRI, the scope of application is

43 limited by the high costs and potential invasiveness of fMRI. To overcome this limitation,

44 several studies adopted EEG, which provides an easy, cheap, and non-invasive way to

45 collect brain activity data. Palazzo et al. (Palazzo et al., 2017) introduced a method for

46 reconstructing the image of an object from EEG signals by converting the EEG signals into

47 features and conditioning generative adversarial networks (GANs) (Goodfellow et al.,

482014 ) by it. This approach allowed them to reconstruct an image that can be correctly

49 classified into the original object category (EEG classification accuracy: 84\%, Inception

50 Score(IS): 5.07, Inception Classification accuracy(IC): 0.43). However, as pointed out by

51 the authors themselves, their result is a product of a generative model conditioned by

52 categorical information extracted from an EEG signal and not the direct reconstruction of

53 the image itself actually given to the observer. It is evident that this method fails to

54 reproduce aspects of the perceptual realism of an image, such as the detailed shape,

55 sharp contours, and textures. This limitation seems unavoidable considering the small

56 data size of EEG signals, especially in terms of spatial resolution. 
57 Against the above background, it is of interest to explore the use of 'texture' images in

58 decoding from EEG signals. The perception of a texture is based on spatially global image

59 statistics (Julesz, 1965; Heeger \& Bergen, 1995; Portilla \& Simoncelli, 2000; Landy \&

60 Graham, 2004; Freeman \& Simoncelli, 2011), and it is even possible to synthesize

61 perceptually similar texture images using only those statistics (Portilla \& Simoncelli,

62 2000). Such statistical information is represented in the low- and mid-level visual cortex,

63 such as V1, V2, and V4 (Freeman et al., 2013; Okazawa et al.,2015; Okazawa et al.,

64 2017; Ziemba et al., 2019), and used in the rapid perception of scenes, objects, and

65 surface materials (Thorpe et al., 1996; Oliva \& Torralba, 2001; Motoyoshi et al., 2007;

66 Rosenholtz et al., 2012; Whitney et al., 2014). In convolutional neural network (CNN),

67 which computationally mimics neural processing in the ventral stream of the visual brain,

68 the spatially global information obtained by the Gram matrix transformation of features

69 extracted from each hierarchical layer stage corresponds to texture representation (Gatys

70 et al., 2015; Gatys et al., 2016).

71 According to these findings, it is expected that texture can be reconstructed from EEG

72 signals by estimating the information that correlates with the spatially global statistics for

73 texture representation. In fact, the recent study (Orima \& Motoyoshi, 2021) were able to

74 estimate lower-order image statistics from VEPs using a linear regression model and

75 synthesize the texture images with identical image statistics. Using the Image-VEP

76 dataset collected in that study, the present paper proposes a CNN-based method that

77 allows a high quality of reconstruction of the original texture image from a VEP for a

78 variety of natural textures.

\section{Method}

80 Texture perception is essentially based on the visual appearance, or impression, of an

81 image according to the continuous perceptual similarity, rather than categorical

82 conceptual knowledge as required for object recognition. From this view, we specifically

83 adopted an MVAE-based approach (Suzuki et al., 2017; Wu \& Goodman, 2018; Kurle et

84 al., 2019; Shi et al, 2019; Tsai et al, 2019) that acquires a continuous latent

85 representation shared by a texture image and EEG signal. Using the trained MVAE model,

86 we attempted to reconstruct the texture image from the latent variables obtained when

87 only one-modality information, EEG data, was input. 
88 In our approach, the MVAE model is trained with the texture images and VEP as two-

89 modality information. After training, the latent space shared by the two modalities is

90 acquired in the model. Finally, the test texture image is reconstructed from the latent

91 variable obtained from the corresponding EEG signals input to the trained model.

\section{EEG measurement}

93 In training the model, we used the dataset obtained by Orima \& Motoyoshi (2021). The 94 dataset comprises EEG signals for 166 natural texture images, with each signal measured 95 for a period of 500 ms, 24 times, for each of 15 human observers.

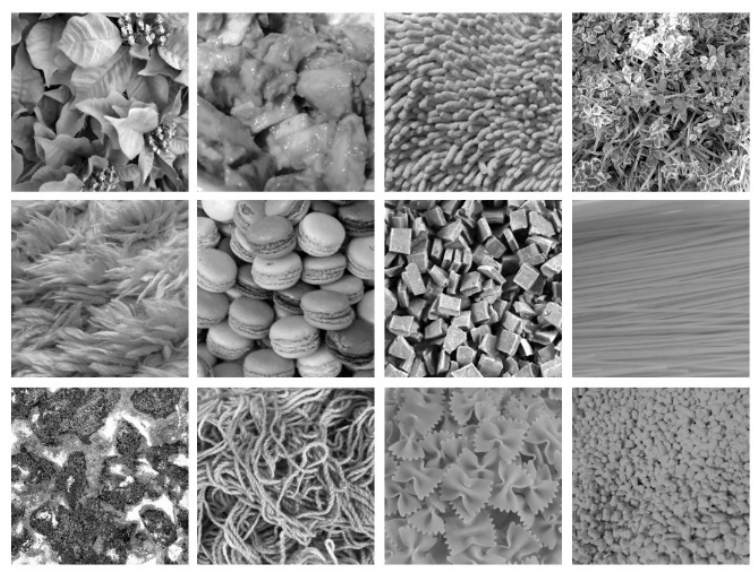

Figure 1. Examples of texture images used in the EEG measurement.

Visual stimuli were images of 166 natural textures subtending 5.7 deg x 5.7 deg ( $256 \times$ 256 pixels). The images were collected from the Internet and our own image database. Each image was achromatic and had a mean luminance of $33 \mathrm{~cd} / \mathrm{m}^{2}$. In each of 24 measurement blocks, 166 images were presented in random order for 500 ms followed by a 750-ms blank that is equal to a uniform gray background and 15 observers viewed each image with their eyes steadily fixed at the center of the image. During each block, the VEP was measured using 19 electrodes (Fp1, Fp2, F3, F4, C3, C4, P3, P4, O1, O2, F7, F8, T7, T8, P7, P8, Fz, Cz, and Pz according to the international 10/20 method; BrainVision Recorder, BrainAmp Amplifier, EasyCap; Brain Products $\mathrm{GmbH}$ ). All stimuli were presented on a gamma-corrected LCD (BENQ XL2420T). The refresh rate of the LCD was $60 \mathrm{~Hz}$, and the spatial resolution was $1.34 \mathrm{~min} /$ pixel at an observation distance of $100 \mathrm{~cm}$. All measurements were conducted in accordance with the Ethics Committee 
110 for Experiments on Humans at the Graduate School of Arts and Sciences, The University

111 of Tokyo. The participants completed a written consent form.

\section{MVAE for image reconstruction from EEG signals}

113 Considering the continuous and variegated nature of natural textures as visual

114 information, we consider a variational auto encoder (VAE) -based (Kingma \& Welling,

115 2013) approach in which the texture images and the corresponding EEG signals are

116 represented in a continuous latent space.

117 The VAE is a deep generative model that conducts its generation process by deep

118 learning assuming the existence of a latent variable $z$ when data $v$ are observed

119 (Kingma \& Welling, 2013; Kingma et al., 2014; Krishnan et al., 2015; Dai et al., 2015).

120 Here, by assuming that latent variables are represented on a probabilistic distribution

121 space, we can perform continuous representation learning on the observed input data

122 (Equation 1).

$$
z \sim p(z)=N(0, I), \quad v \sim p_{\theta}(v \mid z)
$$

124 In the $\mathrm{VAE}$, the observed input data $v$ are transformed by the encoder into a contractive

125 intermediate representation called latent variable $z$, and the decoder reconstructs the

126 original input data $v^{\prime}$ with this latent variable as input. The entire model is trained so as

127 to minimize the difference between the input data $v$ and the reconstructed data $v^{\prime}$, and

128 the model parameters of the encoder and decoder are updated. The encoder and decoder

129 comprise a neural network. (In the following, $\theta$ and $\Phi$ refer to the model parameters of

130 the encoder and decoder, respectively, and the multivariate Gaussian distribution is

131 denoted $\mathrm{p}(\mathrm{z})$.)

132 More practically, the target of training is to maximize the marginal likelihood $p_{\theta}(x)$, but

133 because this cannot be treated directly, we optimize the model parameters of the encoder

$134 q_{\phi}(z \mid x)$ and decoder $p_{\theta}(x \mid z)$ to maximize the evidence lower bound (ELBO) given in

135 Equation 2.

136 In Equation 2, the first term on the right-hand side is called the regularization term. This 137 term regularizes the latent variable $z$, which is obtained basing on the mean vector $\mu$ and 138 variance vector $\sigma$ output by the encoder, to distribute according to prior $p(z)$. 
139 The second term on the right-hand side is the reconstruction error term, which minimizes

140 the difference between the original input data $v$ and $v^{\prime}$, the input data reconstructed

141 from the decoder using the latent variable $z$.

$$
\operatorname{ELBO}(v)=-\beta D_{K L}\left(q_{\phi}(z \mid v) \mid p(z)\right)+E_{q_{\phi}(Z \mid v)}\left[\log p_{\theta}(v \mid z)\right]
$$

143 As an extension of the VAE, the multimodal VAE, which treats multimodal information as 144 input, has been proposed (Suzuki et al., 2017; Wu \& Goodman, 2018; Kurle et al., 2019;

145 Shi et al, 2019; Tsai et al, 2019). This extension is inspired by the fact that our cognition

146 in the real world uses multimodal information, not unimodal information (Ngiam et

147 al.,2011; Srivastava \& Salakhutdinov, 2012; Kiros et al., 2014; Pandey \& Dukkipati,

148 2016). In fact, it is generally known that learning with multimodal information induces the

149 acquisition of better informative representations compared with the case of unimodal

150 information (Ngiam et al.,2011; Srivastava \& Salakhutdinov, 2012).

151 In this study, we apply the extended method for the MVAE (Wu \& Goodman, 2018),

152 which allows inference of latent variables even under the partial observation of

153 multimodal information aiming at reconstructing texture images only from EEG signals.

154 Here, the texture images and EEG signals are treated as different information modalities,

155 and the latent representation shared by these two modalities is acquired by the learning

156 MVAE. As a result of this training, the stimulus can be reconstructed by decoding the

157 texture image using latent variables acquired by the input of a single modality, the EEG

158 signal. Figure 2 is an overview of the structure of the MAVE model. The MVAE model

159 comprises an encoder and decoder for the EEG signal modality and an encoder and

160 decoder for the texture image modality. By inputting one or both of the modalities of

161 information into the encoder corresponding to the respective modal information, a latent

162 variable can be inferred. The latent variables obtained here are integrated into a single

163 latent variable using the product of experts (PoE) (Hinton, 2002). Finally, the

164 reconstructed results of EEG signals and texture images are obtained by inputting this

165 latent variable to each of the decoders corresponding to each modal information. 


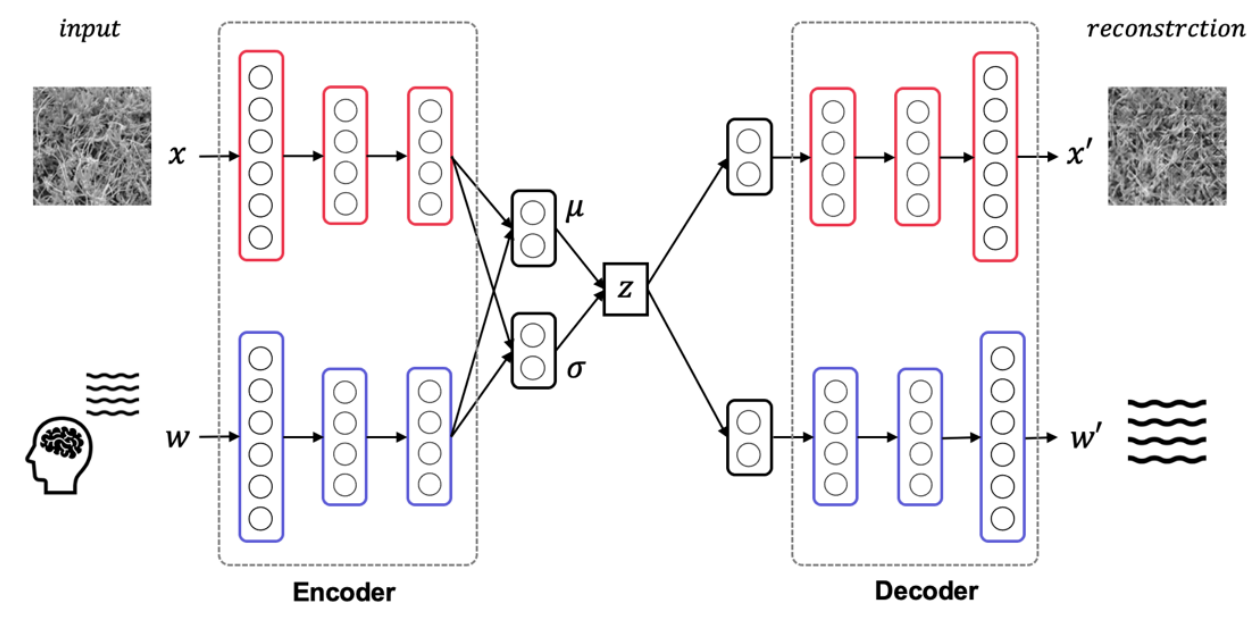

166

167

168

169

170

171

172

173

174

175

176

Figure 2. Model overview. The MVAE model comprises an encoder and decoder for the EEG signal modality and an encoder and decoder for the texture stimulus modality. After training this model, the latent space shared by the two modalities is acquired. Thus, the texture stimulus that was presented in the EEG measurement can be reconstructed from the latent variable obtained by inputting only the EEG signals to this trained model.

In the training of the MVAE model, there are three possible patterns for the combination of the observable modal information. (Here, we denote the texture image modal information as $x$, the EEG signal modal information as $w$, and the observed whole or partial modal information as $\left.V=\left\{v: v_{1}, v_{2}, \ldots v_{n}\right\}\right\}$.)

- $V_{1}=\{v: x, w\}$ : Both modalities, the EEG signal and texture image, can be observed.

- $V_{2}=\{v: x\}:$ One modality, the texture image, can be observed.

$\cdot V_{3}=\{v: w\}:$ One modality, the EEG signal, can be observed.

In reconstructing texture images from EEG signals, which is the target of this study, it is necessary to obtain a representation in the latent space shared by the two modalities of texture images and EEG signals, and to be able to extract latent variables under the partial modal observation $\left(V_{2}, V_{3}\right)$ that are as good as or similar to those extracted under the full modal observation $\left(V_{1}\right)$. Considering this point, we maximize the ELBO expressed in Equation 3, proposed by (Wu \& Goodman, 2018), in our training. 


$$
E L B O(V)=-\beta D_{K L}\left(q_{\phi}(z \mid V) \mid p(z)\right)+E_{q_{\phi}(z \mid V)}\left[\sum_{v_{i} \in V} \lambda_{i} \log p_{\theta}\left(v_{i} \mid z\right)\right]
$$

186 The loss function of the entire model is thus expressed by Equation 4, and the training 187 proceeds accordingly.

$$
\text { loss }=\sum_{V_{i} \in\left\{V_{1}, V_{2}, V_{3}\right\}}-\operatorname{ELBO}\left(V_{i}\right)
$$

One issue that should be considered here is that the image reconstructed using the VAE-

190 based approach is generally blurred. When we tested the reconstruction with the simple

191 VAE using texture images, we found that the reconstruction of fine texture components

192 did not work well, resulting in grayish or blurred images. This is a crucial issue in the

193 present study because we are aiming to realize texture reconstruction with visual

194 similarity to the texture stimuli presented to the observers during EEG measurement. As a 195 solution to such problems, a method combining a VAE and GAN (Rosca et al., 2017) has

196 been proposed to generate natural images and general object images more realistically.

197 However, in the present paper, it is necessary to devise a loss function that improves the

198 reproduction for such texture components when we consider that we use natural texture

199 images in the present study and particularly when failing to reconstruct fine and relatively

200 high spatial frequency components. We thus considered applying precedent knowledge

201 gained in the field of neural style transfer (Gatys et al., 2016; Johnson et al., 2016;

202 Ulyanov et al., 2016; Huang \& Belongie, 2017), where texture synthesis is conducted

203 using trained deep neural network (Gatys et al., 2015). According to this knowledge, in

204 the trained VGG-19 model (Simonyan \& Zisserman, 2015), style information at different

205 levels of abstraction is processed at each stage of the hierarchical processing, and we can

206 extract fine style information at the lower layers and global style information at the higher

207 layers. Additionally, this style information is sufficient for accurate style transferring and

208 texture synthesis (Gatys et al., 2015; Gatys et al., 2016; Johnson et al., 2016; Ulyanov et

209 al., 2016; Huang \& Belongie, 2017). We therefore use this style information in our

210 approach for more precise texture reconstruction. Specifically, we replace the

211 reconstruction error term in the ELBO with a combination of original reconstruction error

212 term and style error term, which is commonly used in the framework of neural style

213 transfer. The style error is expressed in Equation 5. Here, we denote the input image as

$214 x$, reconstructed image as $x^{\prime}$, and set of layers in the trained VGG-19 from which the

215 style information can be extracted as $\mathrm{L}=\{1,2, \ldots \mathrm{k}\}$. Style information obtained by Gram 
216 matrix transformation of the output from each layer is denoted $\mathrm{G}_{\mathrm{X}}=\left\{\mathrm{G}_{\mathrm{x}}^{1}, \mathrm{G}_{\mathrm{x}}^{2}, \ldots, \mathrm{G}_{\mathrm{x}}^{\mathrm{k}}\right\}$ and

$217 \mathrm{G}_{\mathrm{x}^{\prime}}=\left\{\mathrm{G}_{\mathrm{x}^{\prime}}^{1}, \mathrm{G}_{\mathrm{x}^{\prime}}^{2}, \ldots, \mathrm{G}_{\mathrm{x}^{\prime}}^{\mathrm{k}}\right\}$ for the input image and reconstructed image respectively. $\alpha$ is the

218 weighting of style information in each layer, $\mathrm{N}$ is the number of filter maps in each layer

219 of VGG-19, and $\mathrm{M}$ is the number of elements in each filter map in each layer.

$$
\operatorname{StyleLoss}\left(\mathrm{x}, \mathrm{x}^{\prime}\right)=\sum_{\mathrm{l}=1}^{\mathrm{L}} \alpha_{1} \frac{1}{4 \mathrm{~N}_{\mathrm{l}}^{2} \mathrm{M}_{1}^{2}}\left(\mathrm{G}_{\mathrm{x}}^{1}-\mathrm{G}_{\mathrm{x}^{\prime}}^{1}\right)^{2}
$$

221 Applying this style error for the loss function confirmed that the texture pattern can be

222 reconstructed clearly regardless of the spatial frequency of the texture in the input image.

\section{Results}

\section{MVAE model and training}

225 Following the previous EEG decoding study (Palazzo et al., 2017), we used all 166 texture

226 images and corresponding EEG signals in training the model. Instead, for each texture

227 image, the corresponding EEG signals were divided into training, validation, and test

228 datasets according to the proportions of $80 \%, 10 \%$, and $10 \%$ for training the MVAE

229 model. The EEG signals, which were measured for 500 ms after the stimulus onset when

230 the texture stimulus was presented to the observer, were treated as raw vector data with

231500 dimensions. When we input EEG signals to the MVAE model, 25-30 samples of EEG

232 signals corresponding to one particular texture stimuli were selected in random

233 combinations and their average waveforms were normalized in the range of min0 to

234 max1. Among the electrode channels used in the EEG measurement, the signals

235 measured at Fp1, Fp2, F3, F4, C3, C4, P3, P4, O1, O2, F7, F8, T7, T8, P7, and P8 in the

236 international 10/20 method were used as input. Additionally, texture images, as the other

237 information modality, was resized to $128 \times 128$ on input. At this time, the reconstructed

238 texture image was also output as a $128 \times 128$ image. In the training, the Adam gradient

239 descent method was used with a learning rate of $1 \mathrm{e}-4$. The batch size was 16 . The vector

240 size for the latent variable of the MVAE model was 256. The MVAE model comprises an

241 encoder and decoder that treat the texture images as modal information and an encoder

242 and decoder that treat the EEG signals as modal information. We used 2D-convolution for

243 the encoder and decoder that treat the texture images as modal information, and 1D-

244 convolution for the encoder and decoder that treat the EEG data as modal information.

245 The architectural details of the MVAE model are given in Table 1. In actual 
246 implementation, except for the final output layer, each convolution layer is followed by 247 batch normalization and ReLU rectifier processing in order.

\begin{tabular}{|c|c|}
\hline \multicolumn{2}{|c|}{ Texutre-Image Modal } \\
\hline Encoder & Decoder \\
\hline Input 1x128x128 & UpConv2d-3-256 \\
\hline Conv2d-1-32 & UpConv2d-2-128 \\
\hline ResNetBlock-3-32 & ResNetBlock-3-128 \\
\hline AvgPool2d & UpConv2d-4-128 \\
\hline Conv2d-1-64 & ResNetBlock-3-64 \\
\hline ResNetBlock-3-64 & UpConv2d-4-64 \\
\hline Conv2d-1-128 & ResNetBlock-3-32 \\
\hline ResNetBlock-3-128 & UpConv2d-1-32 \\
\hline Conv2d-1-256 & (Sigmoid) \\
\hline $\begin{array}{c}\text { FC-256(mean), } \\
\text { FC-256(var) }\end{array}$ & Output 1x128x128 \\
\hline
\end{tabular}

\begin{tabular}{|c|c|}
\hline \multicolumn{2}{|c|}{ EEG Brain Signal Modal } \\
\hline Encoder & Decoder \\
\hline Input 16x500 & FC-256 \\
\hline Conv1d-3-128-2 & UpConv1d-3-256-1 \\
\hline Conv1d-1-128-1 & UpConv1d-4-128-2 \\
\hline Conv1d-1-128-1 & UpConv1d-4-128-2 \\
\hline Conv1d-1-128-1 & UpConv1d-4-64-2 \\
\hline Conv1d-3-256-2 & UpConv1d-4-64-2 \\
\hline Conv1d-1-256-1 & UpConv1d-4-32-2 \\
\hline Conv1d-1-256-1 & UpConv1d-4-32-2 \\
\hline Conv1d-1-256-1 & UpConv1d-4-1-2 \\
\hline Conv1d-3-512-2 & FC-638 \\
\hline Conv1d-1-512-1 & Output 16x500 \\
\hline Conv1d-1-512-1 & \\
\hline Conv1d-1-512-1 & \\
\hline Conv1d-1-1024-1 & \\
\hline Conv1d-1-256-1 & \\
\hline GlobalAvgPool1d & \\
\hline $\begin{array}{c}\text { FC-256(mean), } \\
\text { FC-256(var) }\end{array}$ \\
\hline
\end{tabular}

Table 1. Details of the model architecture

\section{Reconstruction of the texture image}

251 After training the MVAE model, we reconstructed the texture image using the test EEG 252 signals as input. More specifically, the latent variables were extracted from the encoder 253 that treats the EEG signals as modal information, and the texture images were 254 reconstructed by inputting these latent variables to the other decoder that treats the 255 texture image as modal information. 


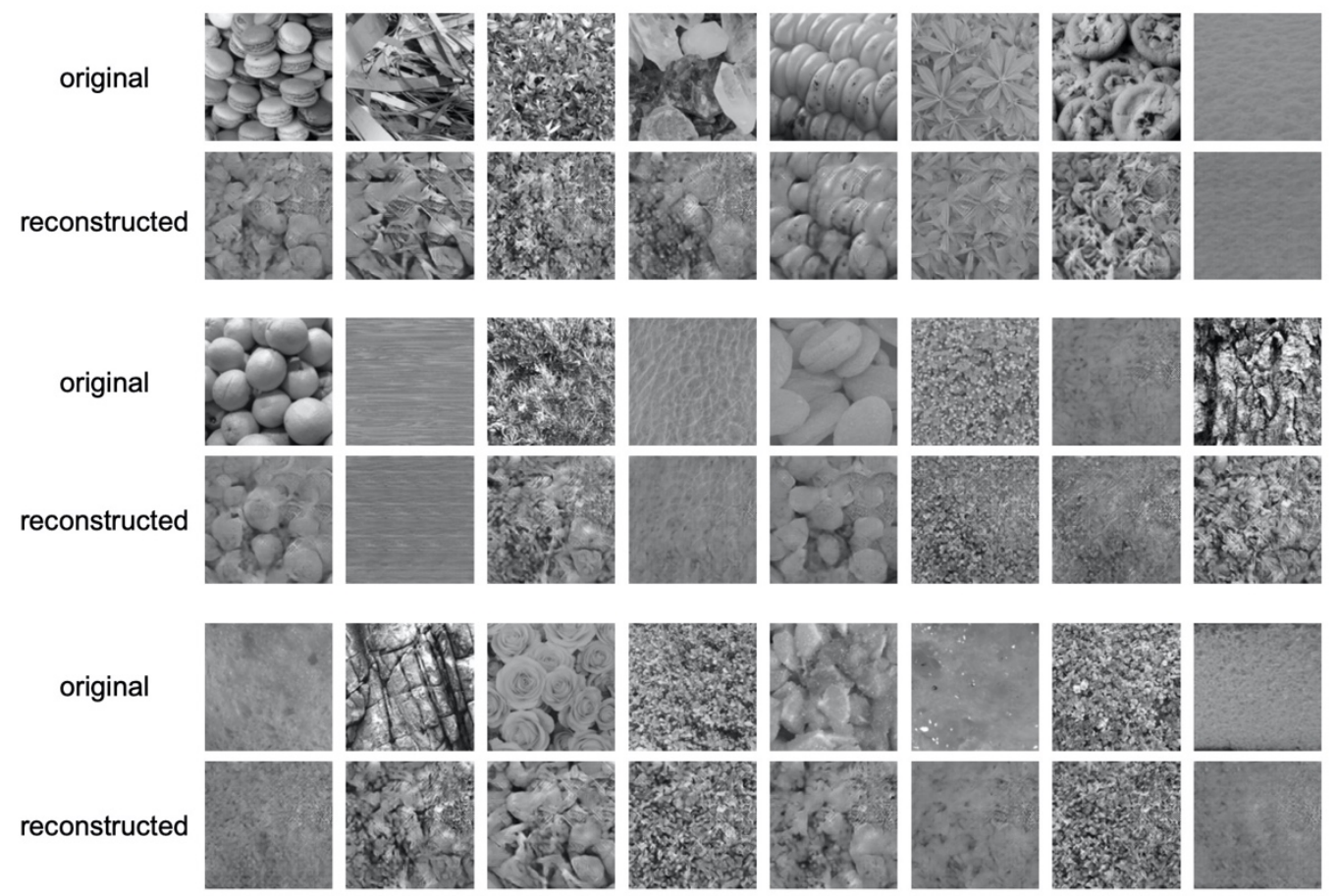

257 Figure 3. Examples of reconstructed texture images. In each row, the upper images are the original
258 texture images shown to the observer and the lower images are the images reconstructed from

259 EEG.

260 Figure 3 shows examples of reconstructed images. In each row, the upper images show

261 the original textures and the lower images show the textures reconstructed from EEG. It

262 is seen that most of the reconstructed textures are remarkably photorealistic, and some

263 are similar to the original textures. The quality of reconstruction is much higher than that

264 of texture synthesis based on linear regression reported in our previous study (Orima \&

265 Motoyoshi, 2021).

\section{Psychophysical experiment}

267 In validating the reconstruction results, we carried out a behavioral experiment to

268 examine the relative perceptual similarity of the reconstructed texture to the original. In

269 our display, the original natural texture $(2.6 \times 2.6 \mathrm{deg}, 128 \times 128$ pixels $)$ was

270 presented at the center, and reconstructed textures were presented on the left and right,

$2713.5 \mathrm{deg}$ from the center. One reconstructed texture was the target image reconstructed

272 from EEG signals for the central original texture, and the other was the non-target image

273 reconstructed from EEG signals for another texture that was chosen randomly from a set 
274 of 165 textures. Six observers with normal or corrected-to-normal vision viewed the

275 stimuli with a free gaze and indicated the texture image (left/right) that was perceptually

276 more similar to the central original texture. To prevent pixelwise matching and the use of

277 memory, each reconstructed texture was randomly chosen from three possible samples.

278 Observers were strongly instructed to evaluate the similarity in terms of the visual

279 appearance and not in terms of the categorical meaning. For each observer, at least four

280 data were collected and the probability of a response that "the target appeared more

281 similar" was calculated for each image. All experiments were conducted using gamma-

282 corrected LCDs with a 60-Hz refresh rate (SONY PVM 2541A, SONY PVM-A250, BenQ

283 XL2730Z, SONY PVM-A250, BENQ XL2720B, and BENQ XL 2430T), each of which was

284 installed in a dark room of the individual observer's home. The viewing distance was

285 adjusted so that the spatial resolution was $1.0 \mathrm{~min} /$ pixel. Other parameters were the

286 same as those in the EEG measurements.

287 Figure 4 shows the probability of a response that "the target appeared more similar"

288 averaged across six observers for 166 textures. The horizontal axis is the index of the

289 texture image, sorted from the left in descending order of the proportion correct. A

290 horizontal red line denotes the chance level (50\%). For $78.1 \%$ of textures, the target

291 image was chosen with a probability significantly higher than the chance level. Together

292 with the observations presented in Figure 3, these results suggest that the reconstruction

293 was fairly successful.

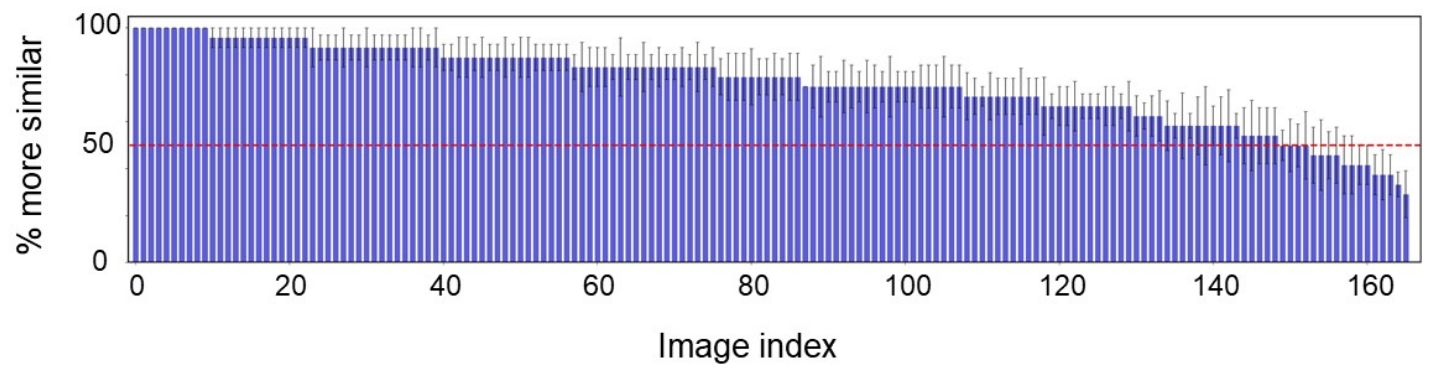

295 Figure 4. Probability of a response that the image reconstructed from the original texture (target)

296 was more similar than the image reconstructed from another random texture (non-target) to the

297 original image. The horizontal axis shows the index of the texture image, sorted from the left in

298 descending order of the probability. The red line denotes the chance level (50\%). The error bars

299 indicate \pm 1 s.e.m. across observers. 


\section{Temporal development of the texture representation}

302 In investigating how the neural representations corresponding to the texture

303 representation that enables such reconstructions evolve over time, we reconstructed

304 texture images from EEG signals with various temporal periods from the stimulus onset

305 and compared their neural representations with those obtained for the entire interval

306 (i.e., $500 \mathrm{~ms}$ ).

307 For this analysis, we prepared 10 samples of EEG signals for each of the 166 original

308 textures of the test dataset used in training the MVAE model. We then input the EEG

309 signals for 100 different temporal periods increasing in length in 5-ms increments from 0

310 ms (i.e., $0-5 \mathrm{~ms}, 0-10 \mathrm{~ms}, \ldots$ 0-500 ms) into the trained MVAE model, and reconstructed

311 the texture image using only the texture representation included in the EEG signal in each

312 period. We denote the image reconstructed from the EEG signal in the entire interval of

$313 \quad 0-500 \mathrm{~ms}$ as $x^{\text {recon }}$ and the texture images reconstructed from the EEG signal in each

314 period as $x_{t=0 \sim 5}^{\text {recon }}, x_{t=0 \sim 10}^{\text {recon }}, \ldots, x_{t=0 \sim 495}^{\text {recon }}$.

315 Figure 5 illustrates the texture images reconstructed with five different temporal periods

316 of the EEG signal. The rightmost image shows the original texture. It is seen that the

317 reconstruction becomes more sophisticated as the temporal period increases.
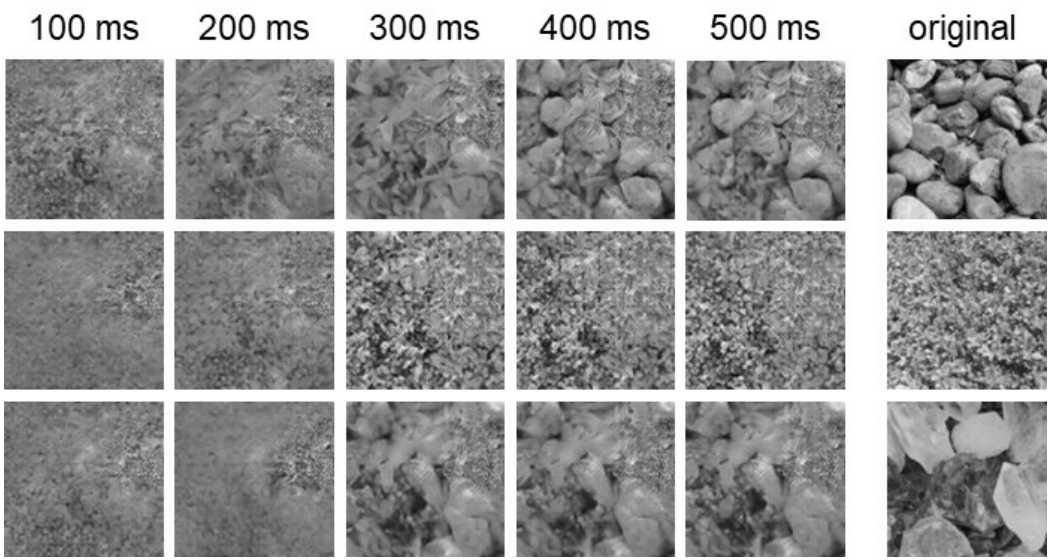

Figure 5. Development of reconstructed texture images over time. In each row, texture images are reconstructed from EEG signals with temporal periods ranging from 100 to $500 \mathrm{~ms}$ (total duration).

321 The rightmost image shows the original texture. 
322 We quantitatively evaluated the reproducibility of texture in the texture images

323 reconstructed from EEG signals in each period using the texture representation metric.

324 (Texture representation is referred to as style information in section 2. Method, but we

325 use the term texture representation in discussing texture here.) Texture representation

326 can be extracted at each layer stage of the trained VGG, which is commonly used in the

327 field of neural style transfer (Equation 5). Here, we input the image reconstructed from

328 the EEG signal in a certain period $x_{t=0 \sim n}^{r e c o n}$ into the trained VGG model and extracted

329 texture representation from the five layers, from the lower layer to upper layer. We

330 conducted the same extraction process for the image reconstructed from the EEG signal

331 including the entire time interval $(0-500 \mathrm{~ms}), x^{\text {recon }}$. The difference in texture

332 representation between $x_{t=0 \sim n}^{\text {recon }}$ and $x^{\text {recon }}$ was then calculated in terms of the root mean

333 square (RMS) for each of the five layers. The calculation was performed for each EEG

334 signal and each period n, which was from 5 to 500 ms. Finally, values were averaged

335 within each layer.

336 Figure 6 plots the development of texture representation over time for each layer of the

337 VGG (denoted by color). The horizontal axis represents the temporal period of the EEG

338 signal, and the vertical axis represents the RMS difference in texture representation

339 between the image reconstructed for that period and the image reconstructed for the

340 entire period (0-500 ms); the difference is normalized to the value for the shortest period

$341(0-5 \mathrm{~ms})$. It is assumed that a smaller relative difference in texture representation

342 indicates better reproducibility of the texture representation. 


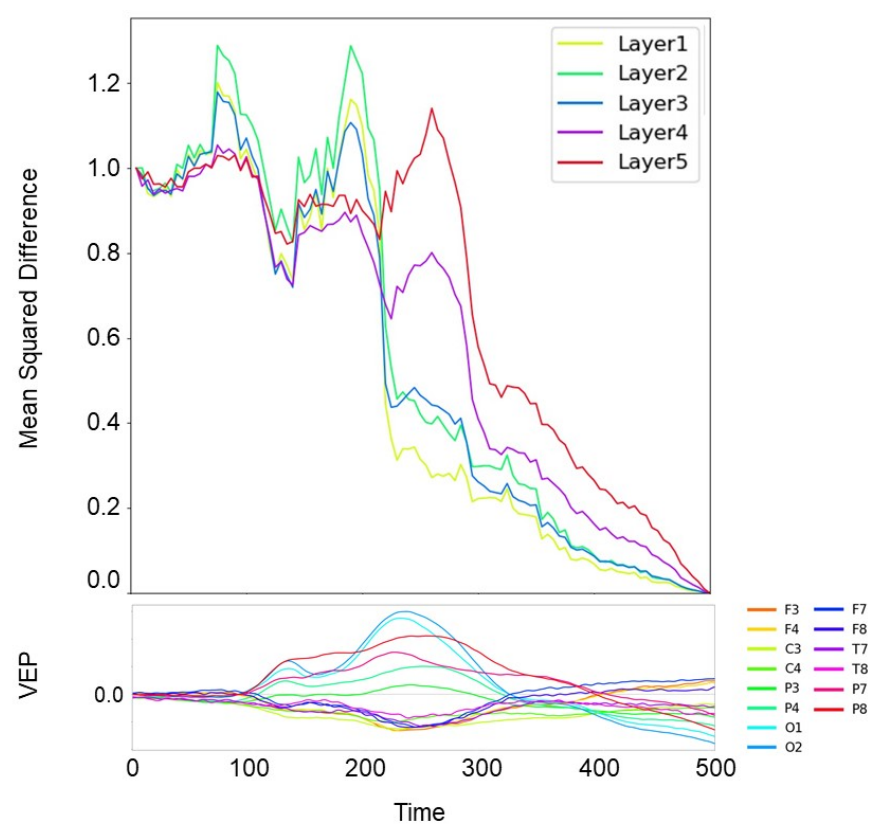

344 Figure 6. Temporal change in the texture representation of the reconstructed image in each layer of the VGG. The horizontal axis represents the temporal period of the EEG, and the vertical axis represents the normalized RMS difference of texture representation from that for the entire period (0-500 ms). Different colors represent the results for different layers of the VGG. The lower panel shows the grand average VEPs for each electrode.

349 The results indicate that the reproducibility of texture representation increases with the 350 temporal period of EEG signals. It is seen that, in the lower layers, namely layers 1, 2,

351 and 3, the reproducibility abruptly increases immediately after $200 \mathrm{~ms}$. This is consistent

352 with the time in which VEPs are correlated with the Portilla-Simoncelli texture statistics

353 (Portilla \& Simoncelli, 2000; Orima \& Motoyoshi, 2021) that are represented in V1 and V2 354 of the primate brain (Freeman et al., 2013). In the higher layers, namely layers 4 and 5, 355 the reproducibility appears to gradually increase especially after $300 \mathrm{~ms}$, suggesting that 356 high-level texture representation develops slowly.

\section{Discussion}

358 The present study introduced a method in which an MVAE is used to reconstruct the 359 image of a natural texture from EEG signals alone. Our trained MVAE model successfully 360 reconstructed the original texture with photorealistic quality and greatly outperformed 361 linear regression on the same dataset (Orima \& Motoyoshi, 2021). Analysis of the 
362 reproducibility of texture representation with temporal accumulation of the EEG signal

363 revealed that the texture representation of the higher layers converges more slowly than

364 that of the lower layers.

365 As mentioned earlier, it is generally challenging to decode neural representations of a 366 natural scene with EEG because of the low retinotopic resolution of EEG as compared with

367 that of fMRI. The present study avoided this limitation by confining the scope to textures

368 to textures for which the perception is determined by spatially global image statistics, and

369 we successfully reconstructed various natural textures from EEG signals. The previous

370 study having a similar scope (Orima \& Motoyoshi, 2021) focused on understanding the

371 neural dynamics for image statistics assumed in human texture perception (e.g., Portilla-

372 Simoncelli statistics) and demonstrated a reconstruction of textures using image statistics

373 linearly regressed from EEG signals. In contrast, the present study pursued a technique to

374 reconstruct an image with higher quality and showed that the use of an MVAE allows the

375 reconstruction of textures with high quality.

376 The previous approach reconstructed natural object images from EEG signals on the basis

377 of the classification of discrete object categories acquired in a supervised network

378 (Palazzo et al., 2017). However, the resulting images did not look naturalistic even

379 though they were classified into the correct object category. In contrast, the present

380 study aimed to reconstruct a purely perceptual impression without any dependency on

381 top-down knowledge such as that of categories, by acquiring a continuous representation

382 space of visual textures in a fully unsupervised learning manner. The resulting images

383 duplicated the perceptual impression well. Of course, such success might be possible only

384 for the textures that we used, and it is unclear if the present approach is applicable to a

385 wide range of classes of images, such as images of objects and scenes. However, we

386 believe that the fact that we were able to reproduce images from EEG signals in a highly

387 realistic manner brings a new direction in the decoding of sensory information. We are

388 currently applying the same approach to sounds.

389 We should also note a limitation of the present approach. Figure 7 shows the worst

390 examples of texture reconstruction. The upper images show the original texture, and the

391 other images show the image samples reconstructed from EEG signals. These

392 reconstructed images are similar to one of the other textures tested, and there is a large 
393 variability among samples for the same original texture. This result is due to the VAE

394 acquiring continuity on visual similarity between the considered textures in the latent

395 space, and therefore, when the proper texture representation was not extracted from the

396 EEG signals, the representation became an intermediate representation that was

397 determined virtually randomly in the space defined by the limited number of textures that

398 we used. As a result, it is highly possible that the reconstructed texture is similar to one

399 of the other original textures. This problem could be avoided with a latent space that is

400 richer with more diverse texture images. However, such a latent space would require

401 many more images and corresponding EEG data.

402

403

404

405

406

407

408

409

410

411

412

413

414

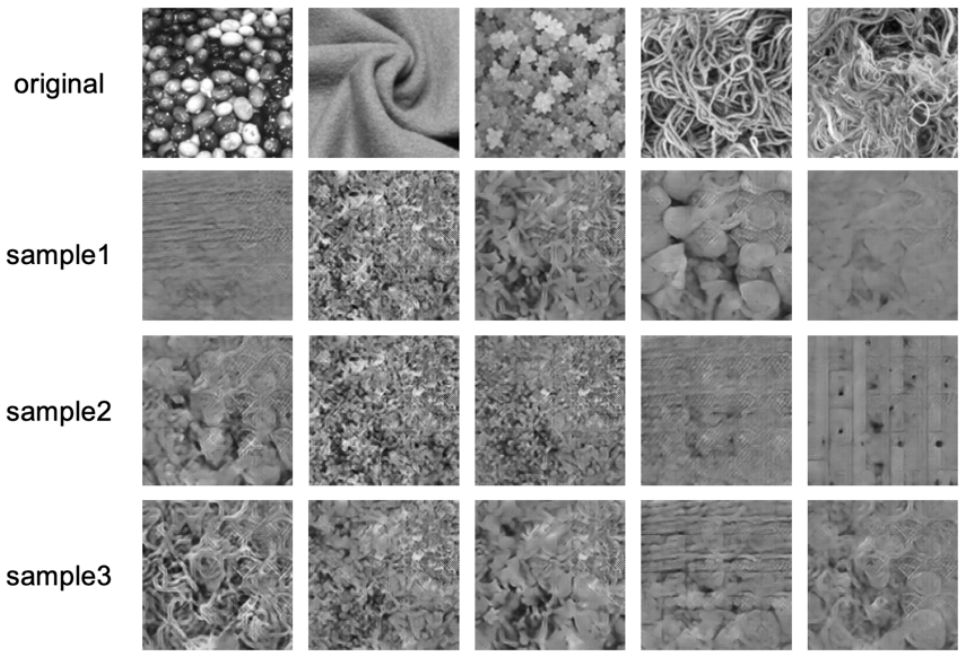

Figure 7. Failure examples of the reconstructed texture images having the lowest 'more similar' response rates in the psychophysical experiment. The upper row shows the original textures and the other rows show three sample images reconstructed from EEG signals.

Analysis on the dynamics of texture representation with temporal accumulation in the EEG signal showed that texture representation becomes sophisticated quickly in the lower layers of the VGG and more slowly in the higher layers of the VGG. In the lower layers, the texture representation converges rapidly from a noisy state around $200 \mathrm{~ms}$ after the stimulus onset. This timing coincides with the temporal epoch (200-250 ms) in which texture VEPs are strongly correlated with Portilla-Simoncelli image statistics especially at high spatial frequencies and with high-level information beyond image statistics (Orima \& Motoyoshi, 2021). In contrast, the texture representation in higher layers converges slowly beyond $300 \mathrm{~ms}$ after the onset. This seems to be related with high-level cortical 
415 representations of global pattern information that integrates the output of the early visual

416 cortex over a large spatial range (Gallant et al., 1993; Schyns \& Oliva, 1994; Okazawa et

417 al., 2015; Portilla \& Simoncelli, 2000; Pasupathy et al., 2020). The other possible

418 interpretation is that this sluggish development reflects changes in neural representation

419 thorough the feedback loop in the visual cortex (Lamme et al., 1998; Bondy et al., 2018;

420 Liang et al., 2017).

421 While the present approach provides an effective tool for reconstructing the visual

422 impression of an image with complex spatial structures from EEG signals, there is still

423 room for improvement. By applying frameworks such as the conditional VAE, the present

424 work is expected to be extended to the analysis of visual impressions specific to a

425 particular individual and the examination of particular visual functions. In the present

426 study, we focused on the pipeline of reconstructing texture stimuli from EEG signals, but

427 owing to the nature of MVAE-based systems, it is also possible to consider the opposite

428 pipeline through which the EEG signal is reconstructed from an image.

\section{Acknowledgment}

430 This study was supported by Commissioned Research of NICT (1940101) and JSPS

431 KAKENHI JP20K21803.

\section{References}

433 Bondy, A. G., Haefner, R. M., \& Cumming, B. G. (2018). Feedback determines the structure of correlated variability in primary visual cortex. Nature Neuroscience, 21(4), 598-606. doi.org/10.1038/s41593-018-0089-1

436 Carlson, T. A., Hogendoorn, H., Kanai, R., Mesik, J. \& Turret, J. (2011). High temporal resolution 437 decoding of object position and category. Journal of Vision, 11(10), 9-9. doi.org/10.1167/11.10.9

438 Carlson, T., Tovar, D. A., Alink, A., \& Kriegeskorte, N. (2013). Representational dynamics of object 439 vision: the first $1000 \mathrm{~ms}$. Journal of Vision, 13(10), 1-1. doi.org/10.1167/13.10.1

440 Dai, Z., Damianou, A., Gonz'alez, J. \& Lawrence, N. (2015). Variational Auto-encoded Deep 441 Gaussian Processes, arXiv preprint arXiv:1511.06455.

442 Das, K., Giesbrecht, B. \& Eckstein, M. P. (2010). Predicting variations of perceptual performance 443 across individuals from neural activity using pattern classifiers. NeuroImage, 51(4), 1425-1437. 444 doi.org/10.1016/j.neuroimage.2010.03.030 
Freeman, J., \& Simoncelli, E. P. (2011). Metamers of the ventral stream. Nature Neuroscience, 14(9), 1195-1201. doi.org/10.1038/nn.2889

Freeman, J., Ziemba, C. M., Heeger, D. J., Simoncelli, E. P. \& Movshon, J. A. (2013). A functional and perceptual signature of the second visual area in primates. Nature Neuroscience, 16(7), 974981. doi.org/10.1038/nn.3402

Gallant, J. L., Braun, J., \& Van Essen, D. C. (1993). Selectivity for polar, hyperbolic, and Cartesian gratings in macaque visual cortex. Science, 259(5091), 100-103.

doi.org/10.1126/science.8418487

Gatys, L., Ecker, A. S., \& Bethge, M. (2015). Texture synthesis using convolutional neural networks. In Advances in neural information processing systems, 28, 262-270.

Gatys, L. A., Ecker, A. S. \& Bethge, M. (2016). Image Style Transfer Using Convolutional Neural Networks. 2016 IEEE Conference on Computer Vision and Pattern Recognition (CVPR). doi.org/10.1109/cvpr.2016.265

Goodfellow, I., Pouget-Abadie, J., Mirza, M., Xu, B., Warde-Farley, D., Ozair, S., Courville, A. \& Bengio, Y. (2014). Generative adversarial nets. In Advances in neural information processing systems, 27.

Green, A. M., \& Kalaska, J. F. (2011). Learning to move machines with the mind. Trends in Neurosciences, 34(2), 61-75. doi.org/10.1016/j.tins.2010.11.003

He, K., Zhang, X., Ren, S. \& Sun, J. (2016). Deep residual learning for image recognition. 2016 IEEE Conference on Computer Vision and Pattern Recognition (CVPR). doi.org/10.1109/cvpr.2016.90

Heeger, D. J., \& Bergen, J. R., (1995). Pyramid-based texture analysis/synthesis. Proceedings of the 22nd annual conference on Computer graphics and interactive techniques - SIGGRAPH ' 95. doi.org/10.1145/218380.218446

Hinton, G. E. (2002). Training products of experts by minimizing contrastive divergence. Neural computation, 14(8), 1771-1800.

Huang, X., \& Belongie, S. (2017). Arbitrary style transfer in real-time with adaptive instance normalization. 2017 IEEE International Conference on Computer Vision (ICCV). doi.org/10.1109/iccv.2017.167

Johnson, J., Alahi, A. \& Li, F. (2016). Perceptual losses for real-time style transfer and superresolution. Computer Vision - ECCV 2016, 694-711. doi.org/10.1007/978-3-319-46475-6_43

Julesz, B. (1965). Texture and visual perception. Scientific American, 212(2), 38-49. doi.org/10.1038/scientificamerican0265-38 
Kamitani, Y,. Tong, F. (2005). Decoding the visual and subjective contents of the human brain. Nature Neuroscience, 8(5), 679-685. doi.org/10.1038/nn1444

Kaneshiro. B., Perreau Guimaraes, M., Kim, H.-S., Norcia, A. M. \& Suppes, P. (2015). A Representational Similarity Analysis of the Dynamics of Object Processing Using Single-Trial EEG Classification. PLOS ONE, 10(8), e0135697. doi.org/10.1371/journal.pone.0135697

Kingma, D. P., Welling, M. (2013). Auto-encoding variational bayes, arXiv preprint arXiv:1312.6114.

Kingma, D. P., Mohamed, S., Rezende, D. J. \& Welling, M. (2014). Semi-supervised learning with deep generative models. In Advances in neural information processing systems, 3581-3589.

Kiros, R., Salakhutdinov, R. \& Zemel, R. (2014). Unifying visual-semantic embeddings with multimodal neural language models. arXiv:1411.2539.

Krishnan, R. G., Shalit, U. \& Sontag, D. (2015). Deep Kalman Filters, arXiv preprint arXiv: 1511.05121.

Krizhevsky, A., Sutskever, I., \& Hinton, G. E. (2012). Imagenet classification with deep convolutional neural networks. In Advances in neural information processing systems, 25, 10971105.

Kurle, R., Günnemann, S., \& Van der Smagt, P. (2019). Multi-source neural variational inference. In Proceedings of the AAAI Conference on Artificial Intelligence, 33(1), 4114-4121.

Lamme, V. A., Super, H., \& Spekreijse, H. (1998). Feedforward, horizontal, and feedback processing in the visual cortex. Current Opinion in Neurobiology, 8(4), 529-535. doi.org/10.1016/s0959-4388(98)80042-1

Landy, M. S., \& Graham, N. (2004). "73 Visual perception of texture," in The Visual Neurosciences, eds L. M. Chalupa and J. S. Werner (Cambridge, Mass: MIT Press), 1106-1118.

Liang, H., Gong, X., Chen, M., Yan, Y., Li, W., \& Gilbert, C. D. (2017). Interactions between feedback and lateral connections in the primary visual cortex. Proceedings of the National Academy of Sciences, 114(32), 8637-8642. doi.org/10.1073/pnas.1706183114

Wu, M., \& Goodman, N. (2018). Multimodal generative models for scalable weakly-supervised learning. In Advances in Neural Information Processing Systems. 5575-5585.

Miyawaki, Y., Uchida, H., Yamashita, O., Sato, M. A., Morito, Y., Tanabe H. C., Sadato, N. \& Kamitani, Y. (2008). Visual image reconstruction from human brain activity using a combination of multiscale local image decoders. Neuron, 60(5), 915-929. doi.org/10.1016/j.neuron.2008.11.004

Motoyoshi, I., Nishida, S., Sharan, L. \& Adelson, E. H., (2007). Image statistics and the perception of surface qualities. Nature, 447(7141), 206-209. doi.org/10.1038/nature05724 
512 Ngiam, J., Khosla, A., Kim, M., Nam, J., Lee, H. \& Ng, A. Y. (2011). Multimodal deep learning. In

513 International Conference on Machine Learning (ICML).

514 Nishimoto, S., Vu, A. T., Naselaris T., Benjamini, Y., Yu, B. \& Gallant, J. L. (2011). Reconstructing

515 visual experiences from brain activity evoked by natural movies. Current Biology, 21(19), 1641-

516 1646. doi.org/10.1016/j.cub.2011.08.031

517 Okazawa, G., Tajima, S., and Komatsu, H. (2015). Image statistics underlying natural texture

518 selectivity of neurons in macaque V4. Proc. Natl. Acad. Sci. U.S.A. 112, E351-E360.

519 doi.org/10.1073/pnas.1415146112

Okazawa, G., Tajima, S., and Komatsu, H. (2017). Gradual development of visual texture-selective properties between macaque areas V2 and V4. Cereb. Cortex 27, 4867-4880.

522 doi.org/10.1093/cercor/bhw282

Oliva, A., \& Torralba, A. (2001). Modeling the shape of the scene: A holistic representation of the spatial envelope. International Journal of Computer Vison (IJCV), 42, 145-175.

Orima, T. \& Motoyoshi, I. (2021). Analysis and synthesis of natural texture perception from visual evoked potentials. Frontiers in Neuroscience, 15, 876. doi.org/10.3389/fnins.2021.698940

Palazzo, S., Spampinato, C., Kavasidis, I., Giordano, D. \& Shah, M. (2017). Generative adversarial networks conditioned by brain signals. 2017 IEEE International Conference on Computer Vision (ICCV). doi.org/10.1109/iccv.2017.369

Pandey, G., \& Dukkipati, A. (2016). Variational methods for Conditional Multimodal Learning:

Pasupathy, A., Popovkina, D. V., \& Kim, T. (2020). Annual Review of Vision Science, 6(1), 363-385.

533 doi.org/10.1146/annurev-vision-030320-041306

Portilla, J., \& Simoncelli, E. P. (2000). A parametric texture model based on joint statistics of 535 complex wavelet coefficients. International Journal of Computer Vision, 40(1), 49-70.

Rosca, M., Lakshminarayanan, B., Warde-Farley, D. \& Mohamed, S. (2017). Variational approaches 537 for auto-encoding generative adversarial networks. arXiv preprint arXiv:1706.04987 
544 Shen, G., Horikawa, T., Majima, K. \& Kamitani, Y. (2019a). Deep image reconstruction from human brain activity. PLOS Computational Biology, 15(1), e1006633.

546 doi.org/10.1371/journal.pcbi.1006633

547

548

549

550

551

552

553

554

555

556

557

558

559

560

561

562

563

564

565

566

567

568

569

570

571

572

573

574

575

576

577

578

Shen, G., Dwivedi, K., Majima, K., Horikawa, T. \& Kamitani, Y. (2019b) End-to-End Deep Image Reconstruction From Human Brain Activity. Front. Comput. Neurosci. 13:21. doi.org/10.3389/fncom.2019.00021

Schwartz, A. B., Cui, X. T., Weber, D. J. \& Moran. D. W. (2006). Brain-controlled interfaces: movement restoration with neural prosthetics. Neuron, 52(1), 205-220. doi.org/10.1016/j.neuron.2006.09.019

Shenoy, P., \& Tan, D. (2008). Human-aided computing: Utilizing implicit human processing to classify images. In CHI 2008 Conference on Human Factors in Computing Systems. doi.org/10.1145/1357054.1357188

Shi, Y., Siddharth, N., Paige, B., \& Torr, P. H. (2019). Variational Mixture-of-Experts Autoencoders for Multi-Modal Deep Generative Models. Advances in Neural Information Processing Systems, 15692-15703.

Simonyan, K., \& Zisserman, A. (2015). Very deep convolutional networks for large-scale image recognition. In Proceedings of the International Conference on Learning Representations (ICLR).

Srivastava, N., \& Salakhutdinov, R. (2012). Multimodal Learning with Deep Boltzmann Machines. In Advances in neural information processing systems, 1,2 .

Stewart, A. X., Nuthmann, A. \& Sanguinetti, G. (2014). Single-trial classification of EEG in a visual object task using ICA and machine learning. Journal of Neuroscience Methods, 228, 1-14. doi.org/10.1016/j.jneumeth.2014.02.014

Suzuki, M., Nakayama, K. \& Matsuo, Y. (2017). Joint multimodal learning with deep generative models. Proceedings of the International Conference on Learning Representations (ICLR) 2017 workshop.

Thorpe, S., Fize, D. \& Marlot, C. (1996). Speed of processing in the human visual system. Nature, 381(6582), 520-522. doi.org/10.1038/381520a0

Tsai, Y. H. H., Liang, P. P., Zadeh, A., Morency, L. P., \& Salakhutdinov, R. (2019). Learning factorized multimodal representations. In International Conference on Learning Representations (ICLR).

Ulyanov, D., Vedaldi, A. \& Lempitsky, V. (2016). Instance normalization: The missing ingredient for fast stylization. arXiv preprint arXiv:1607.08022

Wang, C., Xiong, S., Hu, S., Yao, L. \& Zhang, J. (2012). Combining features from ERP components in single-trial EEG for discriminating four-category visual objects. Journal of Neural Engineering, 9(5), 056013. doi.org/10.1088/1741-2560/9/5/056013 
579 Whitney, D., Haberman, J., and Sweeny, T. D. (2014). "49 From textures to crowds: multiple levels 580 of summary statistical perception," in The New Visual Neurosciences, eds J. S. Werner and L. M.

581 Chalupa (Cambridge, MA: MIT Press), 695-710.

582 Ziemba, C. M., Perez, R. K., Pai, J., Kelly, J. G., Hallum, L. E., Shooner, C., \& Movshon, J. A.

583 (2019). Laminar differences in responses to naturalistic texture in macaque $\mathrm{V} 1$ and $\mathrm{V} 2$. The

584 Journal of Neuroscience, 39(49), 9748-9756. doi.org/10.1523/jneurosci.1743-19.2019 